\title{
Programmauswahl in digitalen Fernsehwelten: Der EPG als Gatekeeper?
}

\author{
Ergebnisse einer Nutzerbefragung
}

\section{Birgit Stark}

Seit über zehn Jabren sind in Deutschland digitale Fernsehangebote empfangbar. Nicht nur die Verspartung der Programme, sondern auch die größere Vielfalt macht die digitale Fernsebwelt für den Zuschauer unübersichtlich. Orientierungshilfe in der nenen Programmfülle soll der Elektronische Programmfübrer (EPG) bieten. Das Spektrum möglicher Funktionen und damit die Auswirkungen auf die Wabrnebmung der Angebote sind groß. Die hier vorgestellte Untersuchung präsentiert EPG-Nutzungsdaten, die im Rabmen einer Nutzerstudie zum digitalen Fernseben aus der Uses-and-GratificationsPerspektive erboben wurden. Mithilfe eines standardisierten Fragebogens wurden rund 600 digitale Fernsehabonnenten eines Kabelnetzbetreibers postalisch interviewt. Die Ergebnisse bestätigen zum einen die Schlüsselfunktion von EPGs im digitalen Fernseben, da eine bobe Nutzungsintensität zu differenzierter Kanalkenntnis und-nutzung fübrt. Zum anderen zeigen sich auch unterschiedliche Verwendungszusammenhänge, die maßgeblich von etablierten Fernsehgewohnheiten und Programmsuchstrategien beeinflusst werden. Alles in allem haben EPGs langfristig das Potenzial, weitaus mebr als nur Bildschirm-Programmzeitschriften zu werden.

Schlagwörter: Digitales Fernsehen, Programmauswahl, Publikumsaktivität, Elektronische Programmführer, Fernsehnutzung

\section{Einführung}

Die Programmfülle via Kabel und Satellit hat in den letzten Jahren enorm zugenommen. Im Durchschnitt kann ein Fernsehhaushalt in Deutschland bereits 55 Sender empfangen. Satelliten-Haushalte haben mit 72 Programmen die größte Auswahl, im Kabel liegt das Angebot bei 45 Sendern, und über Antenne können derzeit durchschnittlich 24 Programme empfangen werden (vgl. Zubayr/Gerhard 2007, 187). Bei den rund 9 Millionen Digitalhaushalten in Deutschland erhöht sich - je nach der Menge der zusätzlich abonnierten Pay-TV-Pakete - die Zahl der Sender nochmals, sodass dem Zuschauer die abendliche Programmentscheidung nicht immer leichtfallen dürfte. ${ }^{1}$ Nicht nur der Markt der klassischen Programmzeitschriften hat sich infolgedessen stark ausdifferenziert, sondern auch gänzlich neue Produkte sind entstanden. Ergänzt werden die traditionellen Printprodukte inzwischen um Online-Services bzw. sogenannte elektronische Programmführer (im Englischen Electronic Programme Guide - EPG). Vor dem Hintergrund der digitalen Programmvervielfachung sollen diese elektronischen Hilfen dem Zuschauer die Orientierung und damit die Programmauswahl erleichtern. EPGs stellen dabei nicht nur Programminformationen zur Verfügung, sie liefern auch funktionalen Mehrwert: Sortierung nach Genre, Zeit oder Sender, Lesezeichen, sendezeitenbezogene Suchanfragen oder die Programmierung einer Schutzfunktion, die ausgewählte Sen-

1 Für das AGF/GfK-Fernsehpanel werden für die Anzahl empfangbarer Programme noch höhere Zahlen ausgewiesen: digital terrestrisch 32, digitales Kabel 94 und digitaler Satellit 101, vgl. Engel 2006, 449. 
dungen für Kinder und Jugendliche verschlüsselt. Durch diese Funktionen bietet der elektronische Programmführer weitaus mehr als die traditionelle Programmzeitschrift. Darüber hinaus könnte der EPG als zukünftiges Einstiegsportal am TV-Gerät ein wichtiger Gatekeeper werden: Elektronische Programmführer können sich nämlich wie ein Filter über Programme und Dienste legen, sodass der Zugriff ausschließlich über die Auswahlregeln des Systems erfolgt.

Angesichts solcher Perspektiven ist es umso erstaunlicher, dass bislang nur wenige empirische Forschungsergebnisse vorliegen, die über die Nutzung elektronischer Programmführer Aufschluss geben. ${ }^{2}$ Die hier vorgestellte Untersuchung will dieses Forschungsdefizit aufarbeiten und präsentiert EPG-Nutzungsdaten, die im Rahmen einer Nutzerstudie zum digitalen Fernsehen aus der Uses-and-Gratifications-Perspektive erhoben wurden. Ziel der vorliegenden Untersuchung soll sein, die Rolle des Elektronischen Programmführers bei der Programmauswahl zu beleuchten. Wird der EPG als Planungshilfe eingesetzt? Wenn ja, welche Funktionen des EPGs werden bereits genutzt? Lassen sich verschiedene Anwendertypen bezüglich verschiedener Such- und Orientierungsstrategien unterscheiden? Welche Auswirkungen hat der Gebrauch des EPGs auf das TV-Nutzungsverhalten und die Kenntnis der Zuschauer über vorhandene Kanaloptionen (Kanalvertrautheit bzw. Kanalrepertoire)? Nach einer kurzen Begriffsbestimmung von EPGs wird ein Überblick über aktuelle Anbieter in Deutschland gegeben, um schließlich auf die Nutzungsaspekte einzugehen. Zentrale Befunde der Studie werden im Abschlusskapitel diskutiert.

\section{Elektronische Navigationshilfen im Fernsehen}

\subsection{Begriffs- und Funktionsbestimmung}

Als Navigationshilfe für den Zuschauer gedacht, soll der Elektronische Programmführer einen Überblick über die Vielzahl an Programmen geben und darüber hinaus durch weitere Funktionen eine individualisierte Fernsehnutzung ermöglichen. Zwei wesentliche Aufgaben können ihm somit zugeordnet werden: Erstens übernimmt er eine „Content“-Funktion, da er eigene medienrelevante Inhalte, Hintergrund- und $\mathrm{Zu}$ satzinformationen zum Programm und damit Verknüpfungsmöglichkeiten zu verwandten Themen oder auch Links zu Online-Diensten anbietet. Zweitens hat er durch den Zugriff zu anderen Kommunikationsinhalten, insbesondere Fernsehprogrammen, eine Steuerungsfunktion. Was herkömmliche Programmzeitschriften in der digitalen Medienvielfalt kaum noch leisten können, übernehmen die Elektronischen Programmführer. Sie ordnen Programminhalte nach bestimmten Genres (Information, Sport, Unterhaltung etc.) und nach speziellen Interessensgebieten. Dabei kommt der Handhabbarkeit der Auswahl- und Filterkriterien des Benutzerführungssystems eine bedeutende Rolle zu (vgl. Leopoldt 2002, 28ff.).

Der Navigator gilt als eine Art Oberbegriff für die verschiedenen Ebenen der Benutzungsführung, des Weiteren kann zwischen dem Basisnavigator, den programmgebun-

2 Auch im Themenheft des „DocuWatch Digitales Fernsehen“ vom Hans-Bredow-Institut findet sich diese Markteinschätzung. Verwiesen wird hier auf einschlägige Untersuchungen im Usability-Bereich, die typisch für diese frühe Phase im Diffusionsprozess sind (vgl. DocuWatch Digitales Fernsehen 2/2005, 17). Zudem wurde im Rahmen der Begleitforschung zur Einführung von DVB-T in Österreich (Graz) eine qualitative Studie durchgeführt (vgl. Paus-Hasebrink 2005). 
denen EPGs einzelner Veranstalter, die dem Zuschauer ausschließlich Informationen über das jeweils eigene Programm liefern, und unabbängigen EPGs von Dritten, die dem Zuschauer programmübergreifende Dienste anbieten, unterschieden werden (vgl. Hasebrink 2001, 55). Als Abgrenzungskriterium der programmgebundenen und unabhängigen EPGs zu den Basisnavigatoren gilt, dass erstere in der Regel nicht die oberste Benutzerfläche repräsentieren, sondern oftmals erst in einem zweiten Nutzungsschritt für den Anwender erreichbar sind (vgl. Leopoldt 2002, 32ff.). Als Einstiegsportal kommt dem Basisnavigator zweifelsohne die größte Bedeutung zu, bereits seine Existenz kann nach Hasebrink $(2001,55)$,zu Veränderungen in der Wahrnehmung des Programmangebots und damit im Suchverhalten und letztlich in der Nutzung führen." Erfolgt beispielsweise der Einstieg direkt über den Basisnavigator, wird der Zuschauer mit der Entscheidung konfrontiert, was er nun auswählen soll und landet nicht bei irgendeinem Programm, wie es bislang der Fall ist (in der Regel je nach Voreinstellung der erste oder der zuletzt eingeschaltete Kanal). Der Stellenwert des bislang sehr bedeutenden Kanalkonzepts dürfte dadurch erheblich geschmälert werden, da der Kanal als Suchkriterium nicht mehr erscheinen muss (vgl. Hasebrink 2001, 54).

Die Elektronischen Programmführer bieten gegenüber den Basisnavigatoren redaktionell gestaltete Programminhalte und weitaus mehr Zusatzinformationen bzw. Softwareanwendungen. Das Spektrum möglicher Funktionen, die EPGs in Zukunft bieten können, ist weit. Neben programmbegleitenden Informationen ist insbesondere die sogenannte „Lesezeichen-Funktion“ relevant. Sie bietet für den Zuschauer die Option, einen Hinweis auf interessante Sendungen zu erhalten, die mit dem Thema der laufenden Sendung verwandt sind. Der Anwender kann hierfür aus einem Stichwortkatalog auswählen und so dem System seine Präferenzen mitteilen, das Gerät weist dann automatisch auf alle aktuellen Sendungen zum Themenschwerpunkt hin. ${ }^{3}$ Diese Zusatzfunktion deutet schon die Weiterentwicklung herkömmlicher EPGs zu sogenannten „intelligenten Navigatoren“ an. Diese ermöglichen eine individualisierte Programmführung und sind in der Lage, Nutzungsprofile einzelner Nutzer zu erstellen, die letztlich auch Rückschlüsse auf persönliche Merkmale ermöglichen. Denkbar ist nicht nur die aktive Programmierung seitens des Nutzers, sondern überdies ein „lernfähiges System“, das dem Nutzer aufgrund bisheriger Sehgewohnheiten Vorschläge unterbreitet.

Derartige Funktionen lassen den Zuschauer also zwischen verschiedenen Nutzungsmodi wählen: Von einer rein rezeptiven Nutzung über die menügesteuerte Suche bis zur Bestellung eines Push-Services (vgl. Hasebrink 2001, 55f.). Während bei der rein rezeptiven Nutzung Sendungen und Programme stärker gewohnheitsmäßig ausgewählt werden, können bei der menügesteuerten Suche über den EPG verschiedene Auswahlmöglichkeiten (Zeit, Kanal, Genre) Hilfestellung leisten. Die Bestellung eines PushDienstes bringt dem Zuschauer darüber hinaus noch für ihn interessante Themen und dies vor allem - sieht man vom ersten Impuls ab - ohne weiteres Engagement.

\subsection{Die wichtigsten Anbieter auf dem deutschen Markt}

In Deutschland haben sich als Anbieter unterschiedliche Akteure etabliert. $\mathrm{Zu}$ nennen sind hier zum einen die Programm- und Netzanbieter und zum anderen Unternehmen aus der Softwarebranche und der Unterhaltungselektronik (z. B. die Hersteller von SetTop-Boxen). Daneben treten auch immer stärker Verlage in Erscheinung. Inzwischen

3 Vgl. zur genauen Funktionsweise Albrecht 1997, 416f. oder auch die Website des Instituts für Rundfunktechnik www.irt.de, Stichwort Electronic Programme Guide und Lesezeichen. 
haben neben dem Pay-TV-Anbieter Premiere alle Kabelnetzbetreiber für die Programmorientierung eigene EPGs entwickelt oder vertreiben diese in Kooperation mit einem Set-Top-Box-Hersteller und vermarkten ihre Programmpakete mit der Box inklusive EPG als Kombilösung. Neben den allgemeinen Programminformationen und verschiedenen Sortierfunktionen bieten die EPGs der Kabelnetzbetreiber spezielle Angebote für die bessere Vermarktung der eigenen Programmpakete und insbesondere zur Nutzung der Near-Video-On-Demand-Angebote. ${ }^{4}$

Auch die öffentlich-rechtlichen Sendeanstalten unterstützen die Vermarktung der digitalen Programmangebote durch eigene EPGs. Allerdings bieten sowohl das ARDPortal als auch der ZDF Digitext ausschließlich Informationen über Sendungen der eigenen Programme. Vergleichbar verhalten sich die privaten Anbieter, beispielsweise RTL, die mit RTL TV Interaktiv über die Angebote des RTL-Bouquets informieren. Noch in der Entwicklung steckt der elektronische Programmguide von ProSieben - bislang ist über das ProSieben iTV-Portal nur wenig bekannt.

Senderübergreifende, redaktionell aufbereitete Informationen bieten insbesondere die speziell entwickelten EPGs großer Zeitschriftenverlage an, die oftmals eine Weiterentwicklung erfolgreicher Printprodukte in elektronischer Form sind. Im direkten Vergleich bieten sie die umfassendsten Informationen für digitale Fernsehzuschauer. Als erster deutscher Verlag etablierte der Heinrich Bauer Verlag einen elektronischen Programmführer: TEO (Television Organizer) startete im Frühsommer 2005 und bietet den Zuschauern aktuelle Programminformationen bis zu 14 Tagen im Voraus. Der Bauer Verlag hat sich außerdem mit einer Applikation von TV MOVIE im Internet etabliert. Das eigenständige Programm ClickFinder lädt das Fernsehprogramm mit dem TV MOVIE-Tagestipp sowie allen Spartentipps, Hintergrundinformationen und Bildern der nächsten Woche auf die Festplatte des Users. Auch der Axel-Springer-Verlag hat das Marktsegment in unterschiedlichen Konstellationen belegt. Das wichtigste Produkt ist die digitale Ausgabe der Fernsehzeitschrift HÖRZU. Neben der einfachen Bedienung bietet der HÖRZU-EPG insbesondere eine Suchfunktion nach Genres (Spielfilm, Doku, Nachrichten etc.) oder nach eigenen Bewertungskriterien der HÖRZU (Spannung, Humor, Action, Anspruch, Erotik).

Die Konkurrenz aus dem Internet ist speziell für Anwender, deren Rechner mit TVKarten ausgestattet sind, interessant. Spezielle Software-Anbieter wie der bereits erwähnte ClickFinder von TV-MOVIE aus der Bauer-Verlagsgruppe oder TVgenial von Arakon Systems helfen, sich sein TV-Programm individuell zusammenzustellen, selbst Videoaufzeichnungen sind programmierbar. Die komplette Programmauswahl ist allerdings nur im Abonnement gewährleistet. Diese kostenpflichtigen Angebote stehen wiederum in Konkurrenz zu sogenannten Open-Source-Programmen, die einen vergleichbaren Service kostenlos anbieten und anderen frei zugänglichen Programmführern mit redaktionell informativ gestalteten Programmen (vgl. Seemann 2005).

Die Auflistung der Anbieter zeigt, dass der Markt in Bewegung ist und insbesondere die großen Zeitschriftenverlage sowohl im Print- als auch im elektronischen Bereich mit entsprechenden Angeboten auf die digitale Programmvervielfachung reagieren. Von Verlagsseite aus werden EPGs (bislang) als Ergänzung zu gedruckten Titeln betrachtet. Insbesondere top-aktuelle Programminformationen sollen am Bildschirm konsumiert,

4 Der Service beinhaltet die zeitversetzte Ausstrahlung eines Spielfilms auf einem oder mehreren Programmen. Im Gegensatz zu sogenannten „True-Video-On-Demand-Angeboten“ kann der Zeitpunkt nicht beliebig gewählt werden, sondern nur in festen Intervallen, beispielsweise zu jeder Viertelstunde (vgl. auch Schenk/Döbler/Stark 2002, 38ff.). 
Hintergrundinformationen hingegen stärker im Printprodukt gesucht werden - so der Gesamtleiter für den Bereich Programmzeitschriften bei Bauer (vgl. Mansmann 2005). Zudem sollen sich die EPGs ganz klar mit ihrem Mehrwert positionieren, insbesondere die Vernetzung und Aufnahmesteuerung mit dem Internet oder per Handy sollen dem Zuschauer helfen, Lieblingssendungen in Zukunft nicht mehr zu verpassen. Der BauerVerlag geht mit seinen Plänen sogar noch weiter. Er arbeitet an einem Programm, das das Sehverhalten der Nutzer analysiert und dem Zuschauer weitere Programmangebote offeriert, die quasi in „freien Zeiten“ genutzt werden können (vgl. Mansmann 2005), ein dem US-Festplattenservice TiVo vergleichbarer Dienst. Das Gerät erkennt die Vorlieben der Nutzer je nach Aufzeichnungsaktivität und archiviert danach automatisch Sendungen, die den Abonnenten interessieren könnten.

\section{Auswahlverhalten im Fernsehen: theoretische Ansätze}

Warum wählen Menschen bestimmte Medienangebote aus und andere nicht? Begibt man sich auf die Suche nach einer Theorie der Mediennutzung, muss man feststellen, dass es keinen umfassenden Ansatz gibt. Die Zuwendung zu Medienangeboten wird nämlich von vielen Faktoren beeinflusst, so dass auch verschiedene Ansätze Erklärungsversuche liefern (vgl. dazu im Überblick Meyen 2004 und Hasebrink 2003). So verwundert es nicht, dass zum einen verschiedene Theorien das Nutzungsverhalten und die Auswahlsituation im Fernsehen beschreiben und zum anderen aber bislang kein Modell existiert, das die sich verändernden Medienumgebungen erschöpfend berücksichtigt. Die vorliegende Untersuchung knüpft theoretisch deshalb an unterschiedliche Ansätze an, Kernpunkt der Arbeit ist jedoch das Konzept der Publikumsaktivität, das im Rahmen der Uses-and-Gratifications-Forschung entwickelt wurde.

Aus dem kommunikationswissenschaftlichen Blickwinkel bezieht sich die Nutzungsforschung schon lange auf den Uses-and-Gratifications-Ansatz und hat diesen auch im Kontext neuer Medien wieder aufleben lassen. Grundgedanke des Erklärungsmodells ist es, dass Rezipienten die sie interessierenden Medieninhalte auf Basis ihrer Motive und Ziele auswählen. Bedürfnisse als Ausgangsbasis sind verantwortlich für bestimmte Erwartungen an die Medien, daraus resultieren konkrete Nutzungsmotive, die letztlich für die Wahl bestimmter Medienangebote ausschlaggebend sind (vgl. zusammenfassend Schenk 2002; Rubin 2002 oder Ruggiero 2000). Eine Adaption auf neue, veränderte Medienumgebungen bietet sich an, da die Forschungsperspektive vom Postulat des aktiven Publikums ausgeht. Die zentralen Annahmen des Ansatzes heben explizit die Bedeutung von Initiative und Aktivität auf Seiten des Publikums hervor, sodass es sich geradezu aufdrängt, die erweiterten Gebrauchsmöglichkeiten infolge der Digitalisierung mit diesem Ansatz zu untersuchen. Zudem stellt er die Komponenten der Medienauswahl in den Mittelpunkt, die von den vielfältigen Neuerungen im digitalen Fernsehen betroffen sind, nämlich Intentionalität und Selektivität. Hierbei handelt es sich um zwei grundlegende Dimensionen im Nutzungsverhalten, die im Rahmen des Konzeptes der Publikumsaktivität bereits eingehend untersucht worden sind und auf die Differenzierungen in den Nutzungsstilen in digitalen Fernsehumgebungen zurückzuführen sind. ${ }^{5}$

Selektivität im Verständnis des Aktivitätskonzeptes (vgl. Levy/Windahl 1984 und

5 Grundlegend für das Modell von Levy und Windahl ist die Annahme von Aktivität als variables Konstrukt, welches in unterschiedlichen Ausprägungen vorliegt. Unterschiedliche Typen und verschiedene Ausmaße von Aktivität variieren in Abhängigkeit von Kommunikationssituationen und -zeiten. 
1985; Blumler 1979) wird dahingehend interpretiert, dass die Auswahl vor dem Hintergrund bisheriger Erfahrungen mit einzelnen Medienangeboten und auch hinsichtlich der jeweils zu erwartenden Gratifikationen für bestimmte Angebote getroffen wird. Intentionalität ist bereits eng verwoben mit dem Begriff der Selektivität und wird als bewusster, absichtsvoller Prozess verstanden, der damit der Selektivität zugrunde liegt. In der konkreten Operationalisierung der Begriffe finden sich in der Uses-and-Gratifications-Forschung allerdings sehr unterschiedliche Bedeutungszuweisungen (vgl. Eilders 1999). Der in der vorliegenden Studie verwendete Selektivitätsbegriff hat mehrere Dimensionen. Zum einen wird Selektivität als inhaltliche Präferenz für bestimmte Genres verstanden, was sich dann beispielsweise in unterschiedlichen Medienorientierungen manifestiert und in der Regel durch unterschiedliche Motive und Einstellungen erklärt wird. Zum anderen wird Selektivität als Auswahlverhalten im Sinne einer Selektionsstrategie interpretiert. ${ }^{6}$ Betrachtet werden Selektionsstrategien sowohl in der präkommunikativen (Grad der Planung) als auch kommunikativen Phase (Umschaltverhalten). Intentionalität wird als Bestandteil des selektiven Fernsehverhaltens in der präkommunikativen Phase eingeordnet und ist damit Teil der Auswahlaktivität. In der empirischen Umsetzung bezieht sich Intentionalität auf die vorherige Planung der Mediennutzung. ${ }^{7}$

Aufgegriffen wurde damit theoretisch nicht nur das Aktivitätskonzept von Levy und Windahl, sondern auch die Vorstellung der differenziellen Aktivität, die mit verschiedenen Medienorientierungen nach Rubin verknüpft werden (vgl. Rubin 1981, 1983 und 1984). Mit der Unterscheidung in instrumentelle, zielorientierte Mediennutzung versus ritualisierte, gewohnheitsmäßige Mediennutzung können unterschiedliche Publika beschrieben werden, deren Medienauswahl durch jeweils unterschiedliche Anstrengungen gekennzeichnet ist. Publikumsmitglieder weisen nicht immer eine selektive und aktive Nutzung auf, häufig werden Medien auch gewohnheitsmäßig, habitualisiert genutzt. Diese gewohnheitsmäßigen Nutzer zeichnen sich durch eine beträchtliche Bindung an bestimmte Medienangebote aus. Aufgrund bereits vorhandener Medienerfahrungen erfolgen viele Auswahlentscheidungen routinemäßig bzw. schematisch. Nicht zuletzt stehen einem notwendigerweise veränderten Medienverhalten damit auch habitualisierte Nutzungsweisen gegenüber, die im Zuge der Programmerweiterung berücksichtigt werden müssen.

Anknüpfend an die Uses-and-Gratifications-Forschung werden zur Erklärung der Fernsehnutzung häufig auch Modelle der Programmauswahl herangezogen. Diese Modelle fügen weitere entscheidende Komponenten ein, die für die Medienauswahl bedeutsam sind, insbesondere die Kenntnis des Angebots (vgl. das integrierte Auswahlmodell in Abbildung 1). ${ }^{8}$ Damit begegnen Programmauswahlmodelle einem der Kritikpunkte

6 Diese zweite Bedeutungszuweisung orientiert sich am traditionellen Verständnis von Selektivität im Sinne von „selective exposure“.

7 Ergänzend wurden zudem die Dimensionen „Involvement“ und „Nützlichkeit“ berücksichtigt, sodass auch Zusammenhänge zwischen den Subkonzepten in den einzelnen Phasen des Kommunikationsprozesses geprüft werden konnten. Auf die letztgenannten Dimensionen soll allerdings im Rahmen der hier vorgestellten Ergebnisse nicht weiter eingegangen werden (vgl. zur Gesamtübersicht der Studie und einer tiefer gehenden Diskussion des Aktivitätskonzeptes Stark 2006).

8 Gemeint ist hier das Modell von Webster und Wakshlag, das alle für die TV-Programmauswahl relevanten Faktoren integriert (vgl. Webster/Wakshlag 1983). Ausgeblendet werden hier die Struktur des Programmangebots bzw. die angewandten Programmierungsstrategien der Sender, da zum einen diese Faktoren nicht in den vorliegenden Forschungsansatz einzugliedern sind 
am Uses-and-Gratifications-Ansatz, der die fehlende Transparenz von Auswahlsituationen bislang theoretisch und empirisch vernachlässigt. Deshalb werden auch in der vorliegenden Studie entsprechende Modelle miteinbezogen, sodass die Analyse der Intentionalitätsdimension unter Berücksichtigung des Programminformationsverhaltens erfolgen kann.

\section{Abbildung 1: Integriertes Gesamtmodell der Medienauswabl}

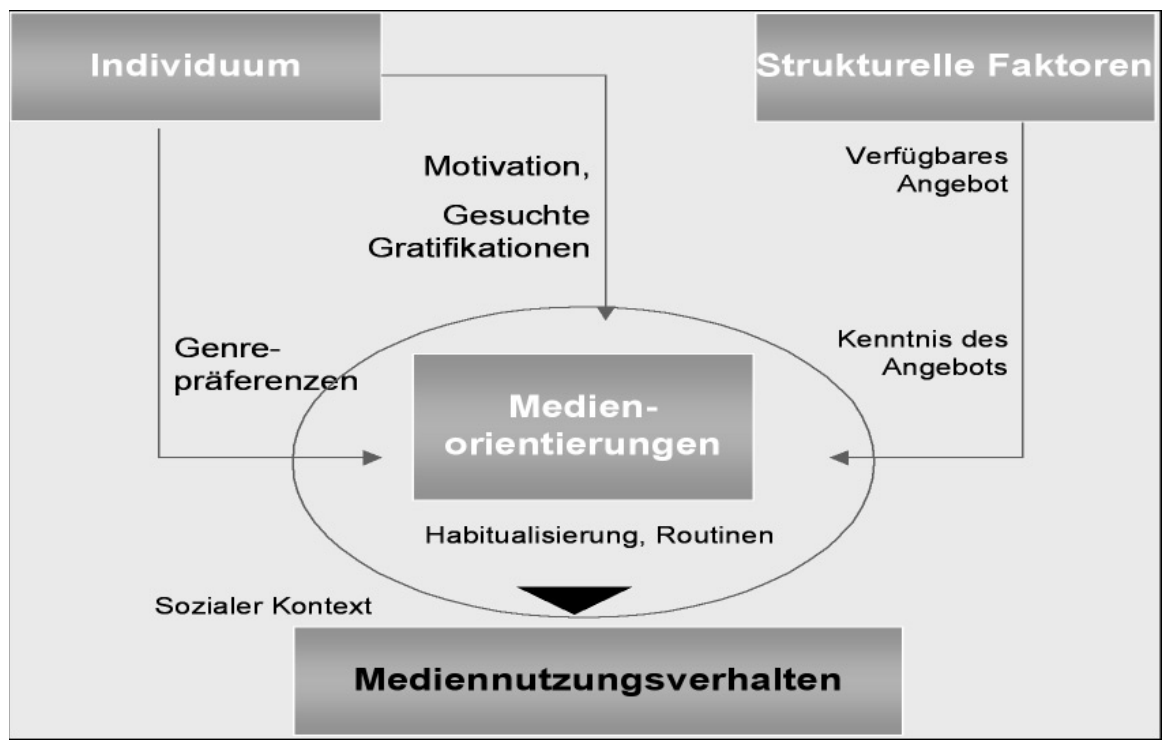

Quelle: Eigene Erstellung in Anlehnung an McQuail 1997, 77.

Insbesondere das Modell von Heeter liefert wichtige Hinweise für ein verändertes Auswahlverhalten in digitalen Fernsehwelten, da es explizit vor dem Hintergrund der Einführung des Kabelfernsehens entwickelt wurde und dem Programminformationsverhalten des Rezipienten eine Schlüsselposition zuordnet (vgl. Heeter 1985 und Heeter/Greenberg 1988). Mit zunehmender Zahl an Programmen muss der Zuschauer erst einmal Kenntnis von allen Programmangeboten erlangen. So konnte Heeter zeigen, dass eine aktivere Orientierung im Entscheidungsprozess die Kanalvertrautheit bzw. das Kanalrepertoire ansteigen lässt. Zu nennen sind hier zum einen die Informationssuche über das Programm vor dem Einschalten des Fernsehgerätes (Planungsphase) und zum anderen die Programmsuche und -auswahl während der Rezeption (Orientierungs- bzw. Wiederbewertungsphase) (Heeter 1985; Ferguson/Perse 1993). Unterschiedliche Aktivitätslevel bei der Programmauswahl können somit Einfluss auf das Kanalrepertoire der Zuschauer haben. Alles in allem finden somit nicht nur Motive als ausschlaggebende Komponente für eine bestimmte Mediennutzung Berücksichtigung, zusätzlich werden auch mögliche Veränderungen in den Teilkonzepten der Zuschaueraktivität überprüft.

Den ersten Hinweis, welche wichtige Rolle der Elektronische Programmführer für

und zum anderen diese in digitalen Fernsehumgebungen an Wirkungskraft verlieren. Methodisch sind hierfür telemetrische Daten notwendig. 
die Auswahlaktivität spielt, liefert eine Untersuchung von Kang (2002). In einer telefonischen Umfrage wurden knapp 400 Abonnenten digitalen Kabelfernsehens zu unterschiedlichen Nutzungsaspekten des EPGs befragt. Erhoben wurden u. a. soziodemographische Variablen, der wahrgenommene Nutzen des EPGs, der Einfluss der Nutzung auf die Höhe des Fernsehkonsums und das Kanalrepertoire. Es stellte sich heraus, dass die Nutzung des EPGs mit einem erhöhten Fernsehkonsum einhergeht und mit einem breiteren Kanalrepertoire verknüpft ist. Dabei erhöhte sich nicht nur die Anzahl, sondern auch die Vielfalt der genutzten Programme. Daneben haben soziodemographische Variablen Einfluss auf den Gebrauch des EPGs: Vor allem jüngere Personen verwendeten den EPG häufiger zur Orientierung. Wenig überraschend spielte der wahrgenommene Nutzen eine Rolle. Sowohl der generell wahrgenommene Nutzen (z. B. Zeitersparnis und Erleichterung bei der Programmsuche) als auch die wahrgenommene Handhabbarkeit beeinflussen das Nutzungsverhalten. Je nützlicher die Befragten die verschiedenen Funktionen fanden, desto häufiger wurde der EPG benutzt, und je komplizierter sie die Bedienung beurteilten, desto häufiger verzichteten sie bei der Programmauswahl auf die Unterstützung durch den EPG. Auch eine längere Erfahrungsdauer mit digitalen Programmangeboten intensivierte den Gebrauch des EPGs. Die Ergebnisse von Kang verweisen bereits auf die zentrale Steuerungsfunktion von EPGs im digitalen Fernsehen. Insbesondere vor dem Hintergrund, dass viele Zuschauer sich ausschließlich in altbewährten Kanalheimaten bewegen und ihre Aufmerksamkeit auf bekannte Kanäle konzentrieren, ihren Kanälen sozusagen „treu bleiben“, wird die Schlüsselfunktion von EPGs deutlich. ${ }^{9}$ Durch ihre Hoheit über Auswahl und Sortierung der verfügbaren Programme beeinflussen sie das „relevant set“ eines digitalen Fernsehzuschauers, weil sie bestehende Kanalloyalitäten aufbrechen und verändern können.

Vor dem Hintergrund der theoretischen Ausführungen gilt es demnach, sowohl den Stellenwert der neu entwickelten elektronischen Programmführer zu beleuchten als auch den Vorgang der Information über das Programm zu betrachten. Wie wird die Fernsehnutzung in Vielkanalwelten geplant? Ein Kanalangebot von 100 und mehr Kanälen steigert den Planungsaufwand, lässt die Programminformiertheit zu einer zentralen Komponente werden und rückt die Intentionalität der Programmauswahl in den Mittelpunkt. Zentrale Forschungsfragen in diesem Zusammenhang sind, ob bestehende Kanalloyalitäten unter dieser Prämisse erhalten bleiben bzw. wie schnell sich das Kanalrepertoire erweitert und den neuen Gegebenheiten anpasst. Außerdem muss davon ausgegangen werden, dass sich eine erhöhte Selektivität in Vielkanalwelten durch eine intensivere Orientierungssuche während der Rezeption auszeichnet, so dass auch das Umschaltverhalten als mögliche Informationsstrategie berücksichtigt wird. Entsprechend liegen dem hier präsentierten Ausschnitt der vorgestellten Studie folgende Fragen zugrunde:

1. Welche Rolle nimmt der EPG in der Planungs- und Orientierungsphase ein? Welche Programminformationsmöglichkeiten werden generell genutzt? Wie häufig kommt der EPG in der kommunikativen Phase zum Einsatz? Welche EPG-Funktionen werden benutzt? Unterstützen die genutzten EPG-Funktionen die bisherige Fokussierung auf das Kanalkonzept? Verändert sich der Umgang mit dem EPG in Abhängigkeit von der Erfahrungsdauer?

2. Welchen Einfluss hat die Auswahlaktivität auf die Kenntnis und Nutzung der digitalen Programmangebote? Wird die Programmauswahl durch den EPG erweitert, weil

9 Als Vereinfachungsstrategie im Vielkanalfernsehen konnte bislang beobachtet werden, dass nicht alle Angebote beachtet bzw. genutzt werden (vgl. Stipp 2003 und Beisch/Engel 2006). 
mithilfe des EPGs erst Programme entdeckt werden können, die ansonsten gar nicht in das Kanalrepertoire mit aufgenommen worden wären? Erhöht sich durch die Nutzung des EPGs die Selektivität der User und erhöht sich somit das Kanalrepertoire? Welchen Einfluss hat eine intensivere Orientierungssuche während der Rezeption? Vergrößert ein höheres Zappinglevel auch das Kanalrepertoire?

\section{Untersuchungsdesign}

\subsection{Stichprobe}

Die empirische Basis für die vorliegende Untersuchung bildet eine postalische Kundenbefragung eines Kabelnetzbetreibers in Deutschland. Die Abonnenten können auf mehr Dienste und Programme zugreifen als die Mehrheit der deutschen Fernsehzuschauer. Kunden, die an das aufgerüstete Breitbandkabelnetz angeschlossen sind, haben Zugang $\mathrm{zu}$ mehr als 100 TV- und Radioprogrammen, zu interaktiver Filmbestellung und zu Highspeed-Internetdiensten. Außerdem können über die Fernbedienung auch interaktive TV-Spiele (z. B. Reversi oder Penalty) gespielt werden, da hier ein „echter Rückkanal“ vom Sender zum Zuschauer existiert. Das digitale TV-Angebot wird in verschiedenen Themenpaketen mit unterschiedlichen Programmschwerpunkten gebündelt. Bereits im Basispaket sind derzeit 45 Programme empfangbar, darunter eine Vielzahl an Spartensendern wie beispielsweise Astro TV, Bibel TV, tv.gusto oder K1010. Ergänzt wird das vielfältige Basisangebot mit Zusatzpaketen zu den Themen Unterhaltung, Trend, Erotik, Kultur, International und Musik. Diese Pakete bewegen sich preislich zwischen 3 und 20 Euro monatlich. In einem Spar-Angebot (Maxi) können mehrere Pakete (Unterhaltung, Trend, Kultur und Erotik) zusammen abonniert werden. Vervollständigt wird das Angebot mit der Option, zusätzliche Filme aus der virtuellen Videothek des Netzbetreibers zu bestellen. Der EPG des Kabelnetzbetreibers ermöglicht eine Durchsicht des kompletten TV-Programmangebotes. Die Auswahl kann entweder nach Uhrzeit, nach Kanal oder auch nach Genre erfolgen. Wenn der Zuschauer einen „Film“ sehen möchte, listet der EPG die Tagesfilme aller Kanäle auf. Auf Wunsch findet man außerdem detaillierte Informationen zu Filmen und Sendungen.

Mithilfe eines standardisierten Fragebogens wurde das Nutzungsverhalten der digitalen Fernsehabonnenten (Ende des Jahres 2002) erforscht. Versendet wurden 1800 Fragebögen, mit einer Rücklaufquote von 35 Prozent bilden rund 600 Fragebögen die Basis für die Auswertung. Die digitalen Fernsehhaushalte sind zum Befragungszeitpunkt vermehrt der Gruppe der Early Adopters zuzuordnen. Dies zeigt sich nicht nur bei der Ausstattung der Haushalte mit elektronischen Geräten der Unterhaltungsindustrie, sondern es verweisen auch bestimmte soziodemographische Merkmale in der Stichprobe (insbesondere Geschlechter-/Altersverteilung und Bildung) darauf. Die Stichprobe zeigt zum einen die Besonderheit, dass Personen männlichen Geschlechts mit 74 Prozent überproportional stark vertreten sind. Zum anderen ist die Altersgruppe der 40bis 49-Jährigen deutlich überproportional vertreten, während die älteren (70 Jahre und mehr) und jüngeren Jahrgänge (14-19 Jahre) schwächer besetzt sind. Auch die Gruppe der höher Gebildeten (Personen mit Abitur (14 \%) bzw. Studium (21 \%)) ist im direkten Vergleich zu allgemeinen Bevölkerungsdaten überproportional stark vertreten, wohingegen die Personengruppe mit Haupt-/Volksschulabschluss mit 17 Prozent seltener ist. ${ }^{10}$

10 Dies zeigt ein Vergleich mit den Strukturdaten der Typologie der Wünsche für Ostdeutschland zum Befragungszeitpunkt (Vgl. Stark 2006, 221ff.). 
Insgesamt deutet die sehr gute technische Ausstattung der Haushalte auf eine besonders hohe Relevanz des Konsums audiovisueller Angebote hin. Im Durchschnitt beziehen die Haushalte seit 19 Monaten digitale Fernsehangebote. Die Abonnementdauer variiert von wenigen Monaten bis zu drei Jahren, wobei knapp zwei Drittel der Abonnenten zwischen einem und zwei Jahren liegen. Länger als zwei Jahre haben 19 Prozent und weniger als ein Jahr 16 Prozent der Kunden digitale Programme abonniert. ${ }^{11}$

Vergleicht man das Programmangebot des Kabelnetzbetreibers zum Befragungszeitpunkt mit dem heutigen Angebot, lassen sich nur geringfügige Veränderungen feststellen. Im Basisangebot sind einige Spartenkanäle durch neue ersetzt worden, die Gesamtzahl der empfangbaren Programme hat sich aber nur gering erhöht. Manche Themenpakete werden inzwischen unter einem anderen Namen vermarktet oder sind in ihrer Zusammenstellung leicht verändert worden, aber die Grundstruktur ist gleich geblieben.

\subsection{Methodisches Design im Fragebogen}

Ein standardisierter Fragebogen erfasste ausführlich das Mediennutzungsverhalten der digitalen Zuschauer und konzentrierte sich entsprechend der theoretischen Ausrichtung auf die Auswahlaktivität vor und während der Rezeption. Schwerpunktmäßig wurde im Rahmen des Aktivitätskonzeptes das Informations- bzw. Auswahlverhalten in der präkommunikativen Phase und das selektive Verhalten in der kommunikativen Phase erfasst. In der Planungs- bzw. Orientierungsphase wurden sowohl die herkömmlichen traditionellen Möglichkeiten (Programmzeitschriften, Videotext, Fernsehprogramm in der Tageszeitung etc.) als Informationsmöglichkeit abgefragt als auch das neue elektronische Medium, der Elektronische Programmführer. Um die individuelle Bedeutung des EPGs für den Fernsehzuschauer zu ermitteln, wurde die Nutzung ausführlich erhoben. Nicht nur die Frage, wie häufig dieser zum Einsatz kommt, war von Interesse, sondern auch die Frage, welche Motive für die Nutzung ausschlaggebend sind. In diesem Kontext wurde zusätzlich die Nutzung der unterschiedlichen Sortierfunktionen abgefragt.

Die Planung der Programmauswahl wurde in einer separaten Frage in Anlehnung an die Intentionalitätsskala von Levy/Windahl behandelt, die in der Originalversion mit drei Items arbeitet. In der Uses-and-Gratifications-Forschung kommen allerdings sehr unterschiedliche Varianten zum Einsatz. Da sich die Items im Original auf bestimmte Programmgenres beziehen, hier aber eine allgemeinere Abfrage erfolgen sollte, wurden in der vorliegenden Studie die Items abgewandelt. ${ }^{12}$ Erhoben wurden folgende Items: TV-Planung am selben Tag bzw. am Anfang der Woche (auch unter dem Aspekt, Lieblingssendungen nicht zu verpassen), spontanes bzw. ungeplantes Fernsehen und Fernsehen als Familienaktivität.

Inwieweit während der kommunikativen Phase eine bereits getroffene Programmentscheidung reevaluiert wird, wurde durch verschiedene Programmauswahlverhaltens-

11 In der Stichprobe befinden sich demnach ausschließlich Zuschauer, denen ein EPG zur Verfügung steht. Eine explizite Kontrollgruppe ohne EPG-Zugang gibt es nicht. Inwieweit sich die digitalen Abonnenten in ihrer soziodemographischen Struktur an „durchschnittliche Fernsehhaushalte" angeglichen haben, kann aufgrund fehlender aktueller Vergleichsdaten leider nicht bestimmt werden.

12 Vgl. Levy/Windahl 1984 bzw. Levy/Windahl 1985. Die Zustimmung bzw. Ablehnung zu den Items wird in der Regel fünfstufig abgefragt. Für die Datenanalyse werden die Items zu einem Index aufsummiert, sodass hohe Werte gleichbedeutend mit einem hohen Intentionalitätsgrad bzw. einer zielgerichteten Planung hinsichtlich der Programmauswahl sind. 
weisen, die im Allgemeinen dem Zappingphänomen zugeordnet werden, unterschieden. Dazu wurden folgende aus der Literatur bekannte Motivationen erhoben: Zapping (Werbevermeidung), Flipping (Überblick über das Programmangebot), Switching (Suche nach weiteren, besseren Angeboten) und Channel Hopping (gleichzeitiges Verfolgen mehrerer Programme). ${ }^{13}$ Die Häufigkeit der Umschaltgründe wurde auf einer vierstufigen Skala erfasst.

Die Liste der bisher verwendeten Motiv-Items in der Uses-and-Gratifications-Forschung ist umfangreich, bis heute hat sich kein Standard durchgesetzt. Die vorhandenen Motivlisten wurden mit wechselnden Items zur Vorhersage einer Vielzahl spezifischer Mediennutzungen eingesetzt. Aus dem Pool der bisher verwendeten Motive in der Uses-and-Gratifications-Forschung wurden die Items so ausgewählt, dass zum einen möglichst vielen Motivaspekten Rechnung getragen werden konnte und zum anderen die von Rubin identifizierten Items für die Differenzierung der unterschiedlichen Medienorientierungen Verwendung fanden. Zudem wurden insbesondere Motivdimensionen ausgewählt, die auf das Fernsehen im Allgemeinen und nicht auf bestimmte Programmgenres anwendbar sind. Zu jeder ausgewählten Motivdimension (Gewohnheit, Zeitvertreib, Geselligkeitsersatz, Unterhaltung, Entspannung, Flucht, Information, soziale Interaktion) wurden mehrere Items formuliert. Die umfassende Motiv-Itembatterie enthielt insgesamt 16 Statements, deren Formulierung sich weitgehend an den einschlägigen Uses-and-Gratifications-Studien orientiert: Zustimmung oder Ablehnung erfolgte mithilfe einer fünfstufigen Likert-Skala.

Das Konstrukt des Kanalrepertoires kann offen, gestützt oder über telemetrische Daten abgefragt werden (vgl. Ferguson/Perse 1993, 32f.). In der vorliegenden Untersuchung wurde die tatsächliche Nutzung der verschiedenen Programme und Programmpakete gestützt mithilfe einer Itemliste abgefragt. Die detaillierte Nutzungsabfrage einzelner Programme beschränkte sich auf die Angebote des Basispakets des Kabelnetzbetreibers, zum Zeitpunkt der Befragung waren im Basispaket 42 Programme enthalten. Die Nutzungshäufigkeit wurde auf einer fünfstufigen Skala erfasst. Zudem wurde erhoben, ob der Sender dem Zuschauer überhaupt bekannt ist. Eine weitere detaillierte Abfrage der Programmangebote aus den sonst noch verfügbaren digitalen Zusatzangeboten war erstens aus Kapazitätsgründen nicht möglich und zweitens den Probanden auch kaum innerhalb einer postalischen Befragung zuzumuten. Insgesamt wurde somit die Nutzung von 42 Programmen detailliert abgefragt, während die Nutzungshäufigkeit bzw. -dauer der acht digitalen Zusatzpakete nur pauschal und nicht auf Programmebene erhoben wurde..$^{14}$

Zusätzlich wurde die sogenannte Fernsehaffinität, also „das Ausmaß der Bedeutung, das die Person dem Medium beimisst“ (vgl. Conway/Rubin 1991, 453), erhoben. In der Regel besteht diese Skala aus fünf Items, die auf einer fünfstufigen Skala bewertet werden. In der vorliegenden Studie wurde die gängige deutschsprachige Version der Fernsehaffinitätsskala nach Rubin eingesetzt. ${ }^{15}$

13 Vgl. beispielsweise Niemeyer/Czycholl 1994, 39ff. Für multivariate Analysen wurde durch Aufsummieren der Werte aller Items ein Index gebildet, der als Maß für die Umschalthäufigkeit (unabhängig von der Motivation) dient.

14 Allerdings wurde für die Zusatzpakete noch die offene Frage nach den Lieblingssendern gestellt. Diese Frage erhebt ungestützt die Präferenzen der digitalen User. Sie gibt zwar keine Auskunft über Nutzungshäufigkeiten, aber zumindest lässt sie Schlussfolgerungen zu, welche Kanäle aus den digitalen Zusatzpaketen sich im „Relevant Set“ der Probanden verankert haben.

15 Vgl. Rubin 1981. Die Skala wurde in unterschiedlichen Stichprobensamples (Kinder, Studieren- 
Erfasst wurden außerdem die üblichen soziodemographischen Merkmale wie Alter, Geschlecht, Haushaltsgröße, Bildung, die Art der Berufstätigkeit, das Einkommen, die Ausstattung des Haushalts mit elektronischen Geräten und die Dauer des Abonnements von digitalen Programmangeboten.

\section{Befunde der Studie}

\subsection{Programminformationsverbalten in der Planungs- und Orientierungsphase}

Zur Frage, welche Informationsmöglichkeiten über das Fernsehprogramm grundsätzlich genutzt werden, entfallen die meisten Nennungen (76 \%) auf die traditionelle Fernseh- und Programmzeitschrift (vgl. Tabelle 1). Hohe Werte erreicht noch der Videotext mit 54 Prozent der Nennungen. Danach folgen „andere Medien“ (beispielsweise das Radio oder das Internet) mit 24 Prozent, das tägliche Fernsehprogramm in der Tageszeitung bzw. die wöchentliche Programmbeilage der Tageszeitung (19 bzw. 17 \%). An letzter Stelle der Nennungen stehen Gespräche mit Freunden oder Bekannten (14\%). Damit bestätigen sich andere Untersuchungen, denen zufolge Programmzeitschriften die wichtigste Informationsquelle für die Programmauswahl der Fernsehzuschauer darstellen. Programmzeitschriften gehören in Deutschland $\mathrm{zu}$ den auflagenstärksten Zeitschriftentiteln. Im internationalen Vergleich nimmt Deutschland hier eine Sonderstellung ein, nirgendwo sonst werden so viele Programmzeitschriften verkauft. ${ }^{16}$ In der vorliegenden Stichprobe zeigen sich zudem deutliche altersspezifische Unterschiede bei der Nutzung traditioneller Informationsmöglichkeiten. So zeichnet sich ein konträres Verhalten zwischen jüngeren und älteren Zuschauergruppen ab. Ältere Personen bevorzugen Printprodukte (z. B. klassische Programmzeitschrift und Tageszeitung), jüngere Fernsehkonsumenten dagegen elektronische Medien (z. B. Radio, Internet oder Videotext) oder auch das Gespräch mit Freunden und Bekannten.

Stellt man diese herkömmlichen Programminformationsmöglichkeiten dem neuen Elektronischen Programmführer gegenüber, lässt sich festhalten, dass die überwiegende Mehrheit der Befragten bereits den EPG benutzt (84\%). Nur eine Minderheit der digitalen Fernsehzuschauer hat bisher weder den EPG des Kabelnetzbetreibers noch den EPG von ARD digital gebraucht (16\%). ${ }^{17}$ Wenig überraschend verzichtet die letztgenannte Gruppe weitgehend auf die Nutzung digitaler Zusatzangebote und beschränkt sich auf das analoge Senderangebot. ${ }^{18}$ So bleibt auch das genutzte Kanalrepertoire unter dem durchschnittlich genutzten (vgl. den nachfolgenden Abschnitt „Einflussgrößen auf das Kanalrepertoire“).

de, Erwachsene, Ältere) vor allem im Rahmen von Uses-and-Gratifications-Studien getestet. Sie gilt als reliabel und valide. Üblicherweise werden die Einzelitems zu einem Index aufsummiert - auch für die vorliegende Analyse ist dies geschehen.

16 Vgl. beispielsweise Hasebrink 2001, 50ff. oder Winterhoff-Spurk 1999, 41.

17 In einigen Fällen sind sich die User unsicher, welchen der beiden EPGs sie benutzen. Allerdings trifft dies nur für einen sehr geringen Prozentsatz zu.

18 Nutzt man das analoge Programmangebot, bedient man sich der herkömmlichen Fernbedienung und verzichtet auf das Einschalten der Set-Top-Box und des EPGs. 
Tabelle 1: Programminformationsverbalten in Abbängigkeit soziodemographischer Merkmale

\begin{tabular}{|c|c|c|c|c|c|c|c|c|c|}
\hline & \multirow[t]{2}{*}{$\begin{array}{l}\text { Ge- } \\
\text { samt }\end{array}$} & \multicolumn{2}{|c|}{ Geschlecht } & \multicolumn{3}{|c|}{ Alter } & \multicolumn{3}{|c|}{ Bildung } \\
\hline & & $\begin{array}{l}\text { Män- } \\
\text { ner }\end{array}$ & $\begin{array}{c}\text { Frau- } \\
\text { en }\end{array}$ & $\begin{array}{c}\text { Bis } \\
30 \mathrm{~J} .\end{array}$ & $\begin{array}{l}30-50 \\
\mathrm{~J} .\end{array}$ & $\begin{array}{l}\text { Über } \\
50 \mathrm{~J} .\end{array}$ & gering & mittel & hoch \\
\hline \multicolumn{10}{|c|}{ Traditionelle Optionen $(\mathrm{n}=626)$ Angaben in Prozent der Befragten } \\
\hline Programmzeitschriften & 76 & 75 & 80 & 64 & 77 & 80 & 83 & 77 & 70 \\
\hline Videotext & 54 & 54 & 54 & 65 & 57 & 45 & 55 & 58 & 48 \\
\hline andere Medien & 24 & 26 & 19 & 46 & 25 & 14 & 19 & 24 & 28 \\
\hline Tageszeitung & 19 & 22 & 12 & 9 & 17 & 27 & 18 & 14 & 26 \\
\hline Supplement Tageszeitung & 17 & 17 & 16 & 11 & 13 & 23 & 18 & 13 & 22 \\
\hline $\begin{array}{l}\text { Gespräche mit Freunden/ } \\
\text { Bekannten }\end{array}$ & 14 & 13 & 19 & 33 & 15 & 6 & 5 & 19 & 12 \\
\hline \multicolumn{10}{|c|}{ EPG-Nutzung $(\mathrm{n}=617)$ Angaben in Prozent der Befragten } \\
\hline gelegentlich / häufig & 60 & 63 & 51 & 67 & 57 & 59 & 68 & 59 & 57 \\
\hline selten & 24 & 24 & 26 & 16 & 27 & 25 & 22 & 25 & 25 \\
\hline nie & 16 & 13 & 23 & 17 & 16 & 15 & 10 & 16 & 19 \\
\hline
\end{tabular}

Anmerkung: Beide Fragen wurden getrennt voneinander abgefragt. Die erste Frage nach den traditionellen Programminformationsquellen wurde als Mehrfachantwortmöglichkeit erhoben. Die zweite Frage erfasste die Nutzungshäufigkeit des EPG auf einer Skala mit den genannten Ausprägungen.

Betrachtet man die EPG-Nutzungshäufigkeit in Abhängigkeit der wichtigsten soziodemographischen Merkmale, finden sich überraschenderweise nur wenige signifikante Unterschiede. So ergeben sich keine signifikanten Alters- oder Bildungsunterschiede - ein Indiz dafür, dass das neue Navigationsinstrument für alle Altersgruppen gleich wichtig ist. Lediglich das Geschlecht hat einen Einfluss auf die Nutzung: Unter den Frauen ist der Anteil der EPG-Nichtnutzer höher (23\%) als unter den Männern (13\%), Frauen verhalten sich insofern skeptischer gegenüber der neuen Technik.

Die Nutzungshäufigkeit des EPGs korreliert hingegen mit der Nutzungshäufigkeit der digitalen Zusatzangebote. Dies gilt für die Zahl der zusätzlich abonnierten Programmpakete, für das Near-Video-On-Demand- und das Spieleangebot. ${ }^{19}$ Dieser $\mathrm{Zu}$ sammenhang ist an sich nicht weiter verwunderlich, da für diese Angebote der Elektronische Programmführer notwendig ist. Zusätzlich wird er aber von dieser Personengruppe nicht nur zur Bestellung weiterer Filme, sondern auch vermehrt zur Planung des Fernsehabends verwendet. Offenbar lässt der häufigere Einsatz der elektronischen Orientierungshilfe Berührungsängste schwinden und die User im Umgang versierter werden. Darüber hinaus gehören die häufigeren EPG-Nutzer vermehrt der Gruppe der Vielseher und gleichzeitig der stark TV-affinen Personen an. ${ }^{20}$

19 In allen Fällen zeigt sich ein geringer Zusammenhang in Höhe von .39 (Anzahl genutzter Zusatzpakete), .39 (Near-Video-On-Demand-Angebote) bzw. .37 (Spielen am TV-Gerät) (Korrelation nach Pearson).

20 Als Wenigseher wurden Personen eingestuft, die bis zu zwei Stunden werktags fernsehen, als Durchschnittsseher Personen, die zwei bis unter vier Stunden fernsehen und als Vielseher wurden Befragte charakterisiert, wenn sie vier und mehr Stunden das Fernsehgerät einschalten. $\mathrm{Da}$ am Wochenende der Fernsehkonsum generell höher ist, wurde hier die Klasseneinteilung leicht verändert: Wenigseher (bis unter drei Stunden), Durchschnittsseher (drei bis unter fünf Stunden) und Vielseher (mehr als fünf Stunden). 
Entgegen den Annahmen zeigen sich bei der EPG-Nutzungshäufigkeit keine signifikanten Unterschiede hinsichtlich der Abonnementdauer. Die einzig feststellbare Tendenz läuft sogar entgegen der Erwartung, dass mit zunehmender Abonnementdauer die Nutzungshäufigkeit des EPGs steigen wird. Es zeigt sich jedoch, dass bei geringer Erfahrung mit digitalen Programmangeboten (unter einem Jahr) das Interesse am EPG größer ist als bei Personen, die bereits länger auf digitale Programmangebote zurückgreifen können. Allerdings verbirgt sich hinter diesem Effekt der Zusammenhang mit der Nutzung digitaler Zusatzangebote: Auch hier nimmt die Nutzungshäufigkeit mit steigender Abonnementlaufzeit ab.

\subsection{EPG-Nutzungsmotive}

Um einschätzen zu können, inwieweit der EPG in Orientierungsstrategien bei der Programmsuche eingebunden ist, wurde nicht nur die Nutzungshäufigkeit abgefragt, sondern auch die unterschiedlichen Nutzungsmotive. Die meisten Nennungen auf diese Frage (79\%) entfallen auf das Item „Ich benutze den EPG, um mich über das laufende Programm zu informieren, indem ich zusätzlich Informationen abrufe“. Am zweithäufigsten (45 \%) wird genannt, dass man sich mithilfe des EPGs über die Filme informiert, die man im Near-Video-On-Demand-Verfahren bestellt. Für eine kleine Nutzergruppe ist der EPG bereits Ersatz für eine normale Fernseh-/Programmzeitschrift. Allerdings muss diese Aussage vorsichtig interpretiert werden, da die Hälfte dieser Personen trotzdem noch angibt, eine Programmzeitschrift zu nutzen. Darüber hinaus wird der EPG auch bereits als Planungshilfe für den Fernsehabend eingesetzt (18\% der Nennungen). Noch weniger Nennungen entfallen auf das Item „Ich habe auch schon Lesezeichen, sogenannte Bookmarks eingegeben ". ${ }^{21}$

Alles in allem hat sich damit der Gebrauch des Elektronischen Programmführers bei der Mehrheit der Befragten etabliert. Allerdings wird dieser eher spontan verwendet, d. h. während des laufenden Programms, sodass die elektronische Programmzeitschrift überwiegend noch eine komplementäre Funktion erfüllt. Nur eine Minderheit setzt den EPG zur Planung des Fernsehabends ein, hier kommen den Befragungsergebnissen zufolge eindeutig stärker traditionelle Informationsmöglichkeiten zum Einsatz. Es zeichnen sich jedoch signifikante altersspezifische Unterschiede beim Gebrauch des EPGs ab. Jüngere Zuschauer unter 30 Jahren setzen den EPG bereits bei mehr Gelegenheiten ein als ältere Zuschauer: Sowohl beim Bestellen zusätzlicher Filme als auch bei der Planung des Fernsehabends wird der EPG stärker von den Jüngeren genutzt, während ältere Personen sich darauf beschränken, den EPG hauptsächlich als Zusatzinformationsmöglichkeit über das laufende Programm zu nutzen. Auch ist der Gebrauch des EPGs für Männer selbstverständlicher als für Frauen, da erstere ihn in mehr Situationen benutzen (vgl. Tabelle 2). Nur wenige der abgefragten Nutzungsmotive zeigen bezüglich der generellen Erfahrungsdauer mit digitalem Fernsehen Unterschiede. Lediglich der Abruf zusätzlicher Informationsmöglichkeiten wird mit zunehmender Abonnementdauer häufiger als Motiv genannt. Andere Funktionen des EPGs sind dagegen explizit mit der Nutzung digitaler Zusatzangebote verknüpft, so dass sich hier die Gebrauchsweisen des EPGs auf

21 Allerdings lässt sich dies mit der Dominanz des Basisnavigators erklären, der die LesezeichenFunktion nicht bietet. Nur wenige kennen und nutzen den programmgebundenen EPG der ARD, der diese Funktion beinhaltet und vom Betriebssystem auch unterstützt werden würde. Ein weiteres Beispiel dafür, wie stark der Einfluss des Basisnavigators sein kann. 
die entsprechende Nutzung der Zusatzoptionen zurückführen lassen (beispielsweise bei der Bestellung zusätzlicher Filme).

\section{Tabelle 2: EPG-Nutzung in Abhängigkeit soziodemographischer Variablen}

\begin{tabular}{|c|c|c|c|c|c|c|c|c|c|}
\hline \multirow[b]{2}{*}{$\begin{array}{l}\text { Angaben in Prozent der } \\
\text { Befragten; Mehrfachnen- } \\
\text { nungen möglich }\end{array}$} & \multirow[t]{2}{*}{$\begin{array}{c}\text { Ge- } \\
\text { samt }\end{array}$} & \multicolumn{2}{|c|}{ Geschlecht } & \multicolumn{3}{|c|}{ Alter } & \multicolumn{3}{|c|}{ Bildung } \\
\hline & & $\begin{array}{c}\text { Män- } \\
\text { ner }\end{array}$ & $\begin{array}{c}\text { Frau- } \\
\text { en }\end{array}$ & $\begin{array}{l}\text { Bis } \\
30 \mathrm{~J} .\end{array}$ & $\begin{array}{l}30-50 \\
\mathrm{~J} .\end{array}$ & $\begin{array}{l}\text { Über } \\
50 \mathrm{~J} .\end{array}$ & gering & mittel & hoch \\
\hline \multicolumn{10}{|c|}{ EPG-Nutzungsmotive $(\mathrm{n}=539)$} \\
\hline \multicolumn{10}{|c|}{ Ich benutze den EPG, um ... } \\
\hline $\begin{array}{l}\text { mich über das laufende } \\
\text { Programm zu informieren, } \\
\text { indem ich zusätzliche In- } \\
\text { formationen abrufe. }\end{array}$ & 79 & 82 & 71 & 77 & 78 & 84 & 82 & 78 & 82 \\
\hline $\begin{array}{l}\text { mich über Filme, die ich } \\
\text { zusätzlich bestellen kann, } \\
\text { zu informieren. }\end{array}$ & 45 & 43 & 50 & 63 & 51 & 30 & 45 & 52 & 36 \\
\hline $\begin{array}{l}\text { Der EPG ist für mich Er- } \\
\text { satz für eine normale Fern- } \\
\text { seh-/Programmzeitschrift. }\end{array}$ & 20 & 20 & 20 & 31 & 21 & 14 & 21 & 18 & 22 \\
\hline $\begin{array}{l}\text { den Fernsehabend zu } \\
\text { planen. }\end{array}$ & 18 & 20 & 13 & 29 & 18 & 15 & 18 & 15 & 24 \\
\hline \multicolumn{10}{|c|}{ EPG-Sortierfunktion $(n=523)$} \\
\hline Uhrzeit & 57 & 58 & 54 & 60 & 58 & 54 & 56 & 58 & 57 \\
\hline Kanal & 50 & 51 & 48 & 53 & 47 & 54 & 58 & 49 & 47 \\
\hline Genre & 37 & 39 & 31 & 31 & 41 & 35 & 32 & 34 & 44 \\
\hline Alphabetische Filmliste & 9 & 7 & 14 & 8 & 10 & 8 & 14 & 10 & 5 \\
\hline
\end{tabular}

Anmerkung: Beide Fragen waren als Mehrfachantwortmöglichkeit konzipiert.

\subsection{EPG-Sortierfunktion}

Wie bereits erwähnt, bietet der EPG verschiedene Sortierfunktionen, die es dem Nutzer auf unterschiedliche Art und Weise ermöglichen, sich im Programmangebot zu orientieren. Bei der Frage, welche dieser Sortierfunktionen benutzt werden, entfallen die meisten Nennungen auf die Funktion Uhrzeit (57 \%), gefolgt von der Kanal- bzw. Genrefunktion (50 \% bzw. 37 \%). Am wenigsten gebrauchen die User die Funktion, die Filme in alphabetischer Reihenfolge anzeigen zu lassen (9\% der Nennungen). Die Tatsache, dass bei der Navigation mit dem EPG neben der „Uhrzeit“ vor allem der „Kanal" benutzt wird, verweist auf die weiterhin bedeutende Rolle der Kanalheimaten trotz des zunehmenden Programmangebots. Da die Information über das laufende Programm an erster Stelle steht, fügt sich der Befund, dass die User mehrheitlich nach Uhrzeit sortieren, in das bereits gewonnene Bild über die EPG-Nutzung ein. Die meisten digitalen User informieren sich vor der Nutzung noch traditionell mit einer Programmzeitschrift, während der Rezeption kommt der EPG dann häufiger zum Einsatz, insbesondere um Zusatzinformationen über das laufende Programm zu erhalten und Reevaluationen des Programms vorzunehmen. Fragen wie „Wann beginnt der nächste Spielfilm?“ oder „Was läuft heute Abend auf meinem Lieblingskanal?" werden dann durch den EPG mit der entsprechenden Sortierfunktion beantwortet. Dabei zeigt sich, dass Wenigseher 
(in der Regel auch weniger stark TV-affine Personen) stärker genreorientiert auswählen als Vielseher. Dieser Nutzungstyp sortiert tendenziell stärker kanalorientiert und benutzt darüber hinaus die alphabetische Filmliste. Die Nutzung der unterschiedlichen Sortierfunktionen verändert sich allerdings nicht in Abhängigkeit von der generellen Erfahrungsdauer mit digitalen Programmangeboten.

Alles in allem zeigt sich, dass die traditionelle Programmzeitschrift nach wie vor einen großen Stellenwert in der Planungsphase einnimmt. Andererseits setzt eine breite Nutzerschaft den Elektronischen Programmführer bereits in der Orientierungs- und Wiederbewertungsphase als Informationsmöglichkeit über das laufende Programm ein, sodass sich hier bereits neue Nutzungsroutinen ausgebildet haben. Die Anwendung dieser Funktion braucht eine gewisse Zeit, um sich zu etablieren. Es zeigt sich nämlich, dass Zuschauer mit einer längeren Erfahrungsdauer (mehr als eineinhalb Jahre) stärker davon Gebrauch machen als Zuschauer, die erst seit kurzem das digitale Fernsehen nutzen (unter einem Jahr).

\subsection{Die Intentionalität der Auswabl von Programmangeboten}

Unterschiedliche Verwendungszusammenhänge lassen sich bezüglich der genutzten Informationsmöglichkeiten und der Programmplanung erkennen. ${ }^{22}$ Zuschauer mit einem hohen Intentionalitätsgrad nutzen bevorzugt die traditionelle Programmzeitschrift, den Videotext oder das tägliche Fernsehprogramm in ihrer Tageszeitung als Informationsmöglichkeit. Zuschauer dagegen, welche die Programmauswahl nicht zielgerichtet vor der Rezeption planen, verzichten in höherem Maße auf die Programmzeitschrift und nutzen stärker Videotext und andere Medien (z. B. das Internet) parallel zur eigentlichen Fernsehnutzung (vgl. Tabelle 3). Während sich der Zusammenhang zwischen dem Intentionalitätsgrad und der Nutzung traditioneller Programmzeitschriften als altersunabhängig erweist, ergeben sich bei den anderen Programminformationsmöglichkeiten in Abhängigkeit vom Grad der Intentionalität Altersunterschiede, wie sie sich bereits generell beim Programminformationsverhalten gezeigt haben. Insgesamt verstärken sich damit die beschriebenen Verwendungszusammenhänge in jüngeren bzw. älteren Altersgruppen.

22 Für die Analyse wurden vier der abgefragten Items in einem ersten Schritt zu einem Intentionalitäts-Index zusammengefasst und in einem zweiten Schritt in drei gleich große Gruppen klassifiziert: geringe Intentionalität (33\% der Befragten), mittlere Intentionalität (34 \%) und hohe Intentionalität (32\%). Nicht mit eingebunden wurden die zwei Items, die stärker situationale Aspekte berücksichtigen und in dem Sinne die individuelle Planung nicht berühren. 
Tabelle 3: Programminformationsverbalten und EPG-Nutzung in Abhängigkeit der Intentionalität

\begin{tabular}{|c|c|c|c|}
\hline \multirow[b]{2}{*}{ Angaben in Prozent der Befragten } & \multicolumn{3}{|c|}{ Grad der Intentionalität } \\
\hline & gering & mittel & hoch \\
\hline \multicolumn{4}{|l|}{ Traditionelle Optionen $(n=615)$} \\
\hline Programmzeitschriften & 55 & 81 & 91 \\
\hline Videotext & 56 & 57 & 49 \\
\hline Andere Medien & 31 & 23 & 18 \\
\hline Tageszeitung & 16 & 17 & 26 \\
\hline Supplement Tageszeitung & 16 & 17 & 18 \\
\hline Gespräche mit Freunden/Bekannten & 16 & 14 & 13 \\
\hline \multicolumn{4}{|l|}{ EPG-Nutzung $(\mathrm{n}=608)$} \\
\hline Gelegentlich/ Häufig & 58 & 55 & 67 \\
\hline Selten & 27 & 28 & 18 \\
\hline $\mathrm{Nie}$ & 15 & 17 & 15 \\
\hline \multicolumn{4}{|l|}{ EPG-Nutzungsmotive $(n=520)$} \\
\hline Abruf laufender Programminfos & 78 & 79 & 82 \\
\hline Infos über Filme im On-Demand Verfahren & 45 & 44 & 46 \\
\hline Ersatz für Programmzeitschrift & 34 & 14 & 13 \\
\hline Planung Fernsehabend & 17 & 18 & 22 \\
\hline \multicolumn{4}{|l|}{ EPG-Sortierfunktionen $(\mathrm{n}=520)$} \\
\hline Uhrzeit & 66 & 54 & 52 \\
\hline Kanal & 44 & 48 & 58 \\
\hline Genre & 37 & 35 & 41 \\
\hline Alphabetische Filmliste & 8 & 10 & 8 \\
\hline
\end{tabular}

Interessanterweise ergeben sich bei der EPG-Nutzungshäufigkeit aber keine signifikanten Differenzen. Tendenziell nutzen zwar Befragte mit hoher Intentionalität bei der Programmauswahl den EPG häufiger als Befragte mit geringer Intentionalität bei der Programmauswahl, wobei die Unterschiede aber gering sind. Ein signifikanter Zusammenhang zeigt sich jedoch in der Art der EPG-Nutzung. Für Personen, welche die Programmauswahl vorab weniger steuern, übernimmt der EPG in größerem Maße eine Ersatzfunktion für die normale Programmzeitschrift, zudem sortieren sie das Programm überwiegend nach Uhrzeit. Im Gegensatz dazu sortieren die Personen, die sich vorab informieren, das Programm mit dem EPG hauptsächlich mithilfe der Kanalfunktion. ${ }^{23}$

Auch hier werden tendenziell die Unterschiede in den Altersgruppen zum einen bestätigt und zum anderen in manchen Fällen sogar verstärkt. Beispielsweise zeigt sich in jüngeren Altersgruppen bei geringerer Intentionalität die Ersatzfunktion bereits häufiger. Eine vorsichtige Interpretation ergibt damit folgendes Bild über mögliche Orientierungs- und Informationsstrategien in digitalen Fernsehwelten:

1. Traditionelle Orientierung: Für eine Zuschauergruppe zeichnet sich eine sehr konservative Informationsstrategie ab. Diese Gruppe steuert die Programmauswahl vor

23 Es zeigen sich in allen Fällen gering ausgeprägte, aber signifikante Korrelationen. 
der Rezeption mit der klassischen Programmzeitschrift und orientiert sich auch bei der EPG-Nutzung stärker am vertrauten Kanalmuster.

2. Elektronische Navigation: Eine andere Nutzergruppe steuert die Programmauswahl weniger zielgerichtet und verzichtet weitgehend auf traditionelle Informationsmöglichkeiten. Als Ersatz hierfür setzt sich in dieser Gruppe der EPG durch. Zudem nutzt dieser Informationstyp auch den Videotext, d. h. die Planungs- bzw. Orientierungsphase fällt in der Regel mit der Rezeptionsphase zusammen.

\subsection{Kenntnis des Angebots und Einflussgrößen auf das Kanalrepertoire}

Die Befunde der vorliegenden Studie zum Kanalrepertoire zeigen sehr deutlich, dass die Anpassung an veränderte Programmwelten bzw. an die steigende Angebotsmenge ein sukzessiver Prozess ist: Die Mehrheit der User nutzt die sogenannte Vereinfachungsstrategie und bewegt sich bislang in etablierten Kanalheimaten, sodass nur ein kleiner Anteil der neuen Spartensender in das persönliche Relevant Set übernommen wird. Im täglich genutzten Kanalrepertoire finden sich durchschnittlich fünf Sender. Erweitert man die Betrachtung auf täglich und mehrmalig in der Woche genutzte Kanäle, sind es im Durchschnitt zwölf von über 40 Kanälen, die im Relevant Set bereits verankert sind. Gleichwohl gelingt es vielen neuen Spartenkanälen nicht, in das persönliche Relevant Set der Zuschauer aufgenommen zu werden - bislang werden die altbekannten Sender (sowohl die Privaten als auch die Angebote der öffentlich-rechtlichen Sendeanstalten) am häufigsten genutzt. Bei einem breiteren Kanalrepertoire finden sich in der Regel aber auch Nachrichten-, Musik- und Dokumentationskanäle (ebenso die digitalen Zusatzangebote der Öffentlich-Rechtlichen). Als größte Hürde erweist sich die mangelnde Kenntnis über das Programmangebot, sodass viele Zuschauer sich aus Unwissenheit über neue Kanäle nur in etablierten, altbekannten „Kanalwelten“ bewegen. Durchschnittlich werden 14 Kanäle überhaupt nicht genutzt. Einige Sender, insbesondere Spartenkanäle aus dem „Very-Special-Interest“-Bereich, sind den Zuschauern überhaupt nicht bekannt.

Bereits in der bivariaten Analyse kristallisieren sich mehrere Einflussfaktoren auf das Kanalrepertoire heraus. Einen starken Einfluss auf die Größe des Kanalrepertoires übt insbesondere die Nutzungsintensität des Fernsehens aus. Hier ergibt sich ein signifikanter Zusammenhang zwischen den einzelnen Sehergruppen und der Anzahl täglich bis mehrmals in der Woche genutzter Sender: Ein überdurchschnittliches Kanalrepertoire haben insbesondere die Vielseher mit einem Mittelwert von 13 Kanälen. Wenigseher liegen dagegen bei nur 10 Kanälen. ${ }^{24}$

24 Beide Variablen korrelieren statistisch signifikant, die Stärke des Zusammenhanges beträgt nach Pearson .24 für die Nutzungsintensität von Montag bis Freitag bzw. .26 am Wochenende. Auch soziodemographische Variablen besitzen Einfluss auf das Kanalrepertoire, allerdings nicht in dem Maße wie die Nutzungsintensität. 
Tabelle 4: Einflussreiche Modellkomponenten auf das Kanalrepertoire ${ }^{25}$

\begin{tabular}{|c|c|c|c|c|}
\hline & Step Entered & $\mathrm{R}^{2}$ & $\mathrm{R}^{2}$-Änderung & Final Beta \\
\hline Soziodemographie & 1 & .01 & .01 & \\
\hline Geschlecht & & & & -.020 \\
\hline Alter & & & & .043 \\
\hline Fernsehnutzung & 2 & $.10 * * *$ & .09 & \\
\hline Fernsehaffinität & & & & .053 \\
\hline Sehdauer & & & & $.205 * * *$ \\
\hline Abonnementdauer & & & & .062 \\
\hline Motivfaktoren $^{26}$ & 3 & $.13 * * *$ & .03 & \\
\hline Zeitvertreib/Ausgleich & & & & -.084 \\
\hline Information/Orientierung & & & & $.109 * *$ \\
\hline Unterhaltung/Entspannung & & & & .057 \\
\hline Aktivitätsdimensionen & 4 & $.17 * * *$ & .04 & \\
\hline Intentionalität & & & & .042 \\
\hline Selektivität (Zapping) & & & & $.198 * * *$ \\
\hline EPG-Nutzungshäufigkeit & 5 & $.22 * * *$ & .05 & $.234 * * *$ \\
\hline
\end{tabular}

In der multivariaten Analyse hingegen zeigt sich nicht nur die Nutzungsintensität als beeinflussende Größe. Entsprechend den Annahmen steuert auch die Auswahlaktivität die Wahrnehmung und Nutzung des Programmangebots. Um den Einfluss auf die abhängige Variable „Kanalrepertoire“ (Anzahl täglich bis mehrmals pro Woche genutzter Kanäle) zu ermitteln, werden - ausgehend von der Prämisse, dass unterschiedliche Aktivitätslevel das Relevant Set der User beeinflussen - sowohl die Motivdimensionen nach Rubin als auch die Subkomponenten des Aktivitätskonzeptes aufgenommen. Zusätzlich werden die wichtigsten Fernsehnutzungsvariablen (Sehdauer, Fernsehaffinität, Abon-

25 Einstellungen bzw. Nutzungshäufigkeiten, die mithilfe einer vier- bzw. fünfstufigen LikertSkala erfasst wurden, werden als intervallskaliert angenommen. Der Test auf Multikollinearität zwischen den unabhängigen Variablen führte zu einem negativen Ergebnis. Betrachtet wurden verschiedene Kollinearitätsmaße (vgl. zu dieser Problematik Brosius 2004, 588f.).

26 Um die verschiedenen Motivdimensionen zu bündeln, wurde eine Faktorenanalyse durchgeführt. Mit der gefundenen 3-Faktorlösung spiegelt sich die Unterscheidung von Rubin in eine instrumentell bzw. ritualisierte Medienorientierung wider. Auf dem ersten Faktor laden Items der Motivdimensionen Gewohnheit, Zeitvertreib, Eskapismus und Geselligkeitsersatz hoch. Der zweite Faktor positioniert Fernsehen dagegen eindeutig als Informations- und Orientierungsmedium: Der Informationsfunktion liegen vor allem Anregungen und Stoff zum Nachdenken und zur Meinungsbildung, Informationen über das Weltgeschehen sowie Lieferung von Gesprächsstoff zugrunde. Der dritte und letzte Faktor wird dagegen durch die Merkmale Spaß, Entspannung und spannende Unterhaltung bestimmt. Während der erste Faktor hauptsächlich einer ritualisiert orientierten Fernsehnutzung und damit einem habituellen Medienkonsum zuzuordnen ist, steht der zweite Faktor „Orientierung“ in erster Linie für eine instrumentell orientierte Fernsehnutzung. Nicht ganz eindeutig positioniert sich dagegen der dritte Faktor, da Entspannung in der Rubin'schen Interpretation überwiegend der ritualisierten Nutzung zugesprochen wird, Unterhaltungsaspekte sich aber sowohl bei dem einen als auch dem anderen Nutzungsmuster finden. 
nementdauer) und die soziodemograpischen Variablen Alter und Geschlecht miteinbezogen. Die Analyse erfolgt mithilfe einer hierarchischen multiplen Regression, bei der die Variablen in aufeinanderfolgenden Blöcken eingegeben werden, deren Reihenfolge vom Anwender theoriegeleitet bestimmt wird. Es kann dann beobachtet werden, wie sich die Ergebnisse der Regressionsanalyse blockweise sukzessive verändern (vgl. Litz 2000, 99ff.). Als Kontrollvariablen werden im ersten Schritt die soziodemographischen Variablen eingefügt, gefolgt von den Fernsehnutzungsvariablen, den Motivfaktoren und den Aktivitätsdimensionen. Die EPG-Nutzungshäufigkeit wurde im letzten Schritt eingefügt.

Alle Variablenblöcke außer dem soziodemographischen zeigen einen statistisch signifikanten Beitrag zur Erklärung des Modells. Die Mediennutzungsvariablen, die im zweiten Schritt beigefügt wurden, erhöhen den Anteil der erklärten Varianz um 9 Prozent, gefolgt von 3 Prozent durch die Motivfaktoren. Die Aktivitätsdimensionen im vierten Schritt erhöhen die erklärte Varianz um 4 Prozent und die EPG-Nutzung im letzten Schritt um 5 Prozent.

Als signifikante Einflussgrößen mit einem hohen Erklärungsbeitrag erweisen sich nach dem letzten Schritt die EPG-Nutzungshäufigkeit und die Umschalthäufigkeit während der Rezeption: Wie erwartet, vergrößert sich das Kanalrepertoire, je häufiger der EPG genutzt wird. Zudem zeigt sich, dass vermehrtes „Surfen“ in der Orientierungs- bzw. Wiederbewertungsphase die Bandbreite häufig genutzter Kanäle erhöht. Dagegen hat das Ausmaß der Planung der Fernsehnutzung keinen Einfluss auf das Kanalrepertoire, d. h. die Zielgerichtetheit der Programmauswahl führt im digitalen Fernsehen nicht zu einer erweiterten Wahrnehmung der Programmalternativen. Damit stehen die hier gewonnenen Befunde im Gegensatz zur Studie von Ferguson und Perse. Eine mögliche Erklärung für diese widersprüchlichen Befunde könnte darin liegen, dass Ferguson und Perse diesen Zusammenhang lediglich mit dem sogenannten „mindful channel repertoire“ (ungestützte Erfassung der in der letzten Woche genutzten Kanäle) herstellen konnten. ${ }^{27}$ In der hier durchgeführten Abonnentenbefragung wurde die Nutzung der Kanäle aber gestützt erhoben.

Während sich in der genannten Studie die Fernsehnutzungsmotive als nicht signifikant für das "mindful channel repertoire" erwiesen, zeigen sich in der vorliegenden Untersuchung Einflüsse dieses Faktors. Der stärker habituell geprägte Motivfaktor „Zeitvertreib, Ausgleich und Flucht“ ist mit dem Kanalrepertoire negativ verknüpft (allerdings mit $\mathrm{p}=.07$ nicht mehr signifikant). Zuschauer mit einem starken Informationsbzw. Orientierungsbedürfnis zeigen sich dagegen aufgeschlossener und nutzen mehr Kanäle. Dies entspricht den Grundannahmen unterschiedlicher Medienorientierungen, die die instrumentelle Nutzung als weitaus aktiver interpretiert. Der Unterhaltungsfaktor jedoch zeigt keinerlei Wirkung. Entgegen den Annahmen erweist sich die Bindung an das Medium im Regressionsmodell nicht mehr signifikant, obwohl dieses Merkmal sich in der bivariaten Analyse als Einflussgröße auf das Kanalrepertoire herauskristallisiert hat. Desgleichen hat sich die Erwartung, dass mit zunehmender digitaler Fernseherfahrung das Kanalrepertoire steigt, nicht erfüllt: Das Merkmal „Abonnementdauer“ erwies sich als nicht signifikant.

Alles in allem kristallisieren sich folgende Verhaltensweisen als relevante Größen für das Kanalrepertoire heraus: eine hohe Nutzungsintensität und der häufige Gebrauch der Fernbedienung, sei es um den EPG zu aktivieren oder um durch die Programme zu

27 Vgl. Ferguson/Perse 1993, 40ff. In den Studien von Heeter und Greenberg (1988) zeigte sich dagegen auch kein Zusammenhang zwischen Programmplanung und Kanalrepertoire. 
zappen. Alle genannten Merkmale führen sowohl zu differenzierter Kanalkenntnis als auch Kanalnutzung.

\section{Zusammenfassung und Schlussfolgerungen}

Einen Überblick über das stark verzweigte Programmangebot im digitalen Fernsehen verschafft sich die Mehrheit der Zuschauer noch traditionell mithilfe der klassischen Programmzeitschrift, gefolgt vom Videotext und der Option, andere Medien wie Radio oder Internet als Informationsquelle zu nutzen. Die elektronische Informationsmöglichkeit steht der klassischen Programmzeitschrift aber nicht nach: Nur eine kleine Minderheit verweigert gänzlich die EPG-Nutzung; gegenwärtig findet sich in dieser Gruppe noch ein höherer Frauenanteil. Der vermutete Effekt auf das Kanalrepertoire hat sich bestätigt: Die Nutzung des EPGs erhöht das Kanalrepertoire, sodass intensive EPG-Nutzer über ein überdurchschnittliches Kanalrepertoire verfügen. Dabei korreliert die EPG-Nutzungshäufigkeit erwartungsgemäß mit der Nutzungshäufigkeit digitaler Zusatzangebote. Interessanterweise zeigt diese aktivere Nutzergruppe aber auch ein breiteres Anwendungsfeld, sodass der EPG hier nicht nur als Informationsquelle zum laufenden Programm genutzt, sondern verstärkt zur Planung des Fernsehabends eingesetzt wird. Alles in allem hebt sich hier bereits eine jüngere, mehrheitlich männliche Anwendergruppe ab, die sich im Umgang mit den digitalen Zusatzangeboten und mit dem EPG versierter zeigt. Dagegen ergeben sich aufgrund der Erfahrungsdauer bislang nur wenige Nutzungsdifferenzen. Jedoch prägen sich bestimmte Nutzungsroutinen erst im Laufe der Zeit aus, beispielsweise der Abruf zusätzlicher Informationen über das laufende Programm.

Die am häufigsten benutzten EPG-Sortierfunktionen dokumentieren, welche Funktionen der EPG für die Zuschauer erfüllt. Die menügesteuerte Suche erfolgt am häufigsten über die Uhrzeit, gefolgt von der Kanal- und Genrefunktion. Am seltensten wird die alphabetische Filmliste benutzt. Dabei liegen Uhrzeit und Kanal bei den Nennungen dicht beisammen, ein weiteres Indiz dafür, dass Kanalheimaten nach wie vor eine hohe Bedeutung haben. Eine Programmierung des Systems in Form von Lesezeichen findet bislang kaum statt. Hier zeigt sich, dass noch wenig Bereitschaft besteht, die rein rezeptive Nutzung bzw. die menügesteuerte Suche zu einem Push-Service zu erweitern.

In zweierlei Hinsicht hat sich in der vorliegenden Studie bestätigt, dass die Auswahlaktivität die Kenntnis über Programmangebote beeinflusst. Zum einen hat sich eindeutig gezeigt, dass die Zuschauergruppe, die sich während der Rezeption selektiver verhält und häufiger umschaltet, mehr Kanäle kennt und nutzt. Eine verstärkte Orientierungssuche während der Rezeption ist also positiv mit dem Kanalrepertoire verknüpft. Zum anderen hat sich die Schlüsselfunktion des EPGs bestätigt, da auch er die Wahrnehmung und Nutzung des Angebots beeinflusst. Im Durchschnitt sind es in der Studie zwei bis drei Kanäle mehr, die durch diese Verhaltensweisen in das regelmäßig genutzte Kanalrepertoire aufgenommen werden.

Die Ergebnisse der Untersuchung bestätigen unterschiedliche Verwendungszusammenhänge von EPGs, vergleichbar zu analogen Programmwelten, in denen bestimmte Fernsehgewohnheiten den Umgang mit Programmzeitschriften steuern. Es scheint, dass sich etablierte Fernsehgewohnheiten in digitalen Programmwelten weiterentwickeln, aber durchaus auf unterschiedlichen Ebenen. Zuschauer, die ihre Fernsehnutzung „konservativ" planen und hierfür bislang auf traditionelle Informationsmöglichkeiten zugegriffen haben, setzen den EPG auch eher herkömmlich im Sinne des Kanalkonzeptes ein. Dagegen zeichnet sich eine Zuschauergruppe ab, die viel stärker „neue Medien“ zur 
Programminformation nutzt und daher konsequent den EPG stärker einbindet. Fernsehzuschauer, die an die Logik einer PC-Benutzeroberfläche gewöhnt sind, integrieren das neue Suchsystem schneller und nutzen infolgedessen andere und mehr Suchfunktionen. Die Forschung in den USA zeigt, dass der EPG dort in vielen Haushalten bereits die Programmzeitschrift als Informationsquelle ersetzt (vgl. Stipp 2004, 571). Gerade PC-affine, junge Zuschauer verzichten immer häufiger auf die Nutzung von Programmzeitschriften (vgl. Stipp 2003, 474).

Im direkten Vergleich steht Deutschland erst am Beginn einer Entwicklung, deren weiterer Verlauf bedingt durch unterschiedliche Anbieterinteressen noch nicht absehbar ist. Rund ein Viertel der Fernsehhaushalte hat Zugang zum digitalen Fernsehen und bietet sich als potenzielle Zielgruppe für die Nutzung elektronischer Programmführer an. Der Marktüberblick zeigt, dass sich viele Systeme noch in der Entwicklung befinden, und derzeit noch völlig offen ist, ob sich die EPGs der Verlage, der Content-Anbieter oder der Provider auf breiter Front durchsetzen werden. Auch die Navigationsfunktionen und deren unterschiedliche Ausgestaltungsmöglichkeiten werden noch getestet, sodass bislang nicht geklärt ist, mit welcher Auswahlsituation die Fernsehzuschauer konfrontiert sein werden, wenn der digitale Umstieg wirklich vollzogen wird. Als Einstiegsportal in die digitale Fernsehwelt wird der EPG aber zukünftig Selektionsentscheidungen mitgestalten können. Die Dominanz seines Einflusses wird zum einen stark vom Aufbau und der Beschaffenheit der Benutzeroberfläche abhängig sein und zum anderen von fest etablierten Fernsehgewohnheiten gelenkt werden. Es deutet sich an, dass verschiedene Nutzertypen die EPGs unterschiedlich schnell und umfangreich in ihr Auswahlverhalten integrieren. Dabei spielen bestehende Programmsuchstrategien eine entscheidende Rolle; die orientieren sich bislang noch stärker an einer „rezeptiven Nutzung“. Wie schnell diese an digitale Gegebenheiten angepasst werden, wird sich zeigen müssen. Das bisherige Nutzerverhalten - auch bezüglich des Kanalrepertoires - deutet auf einen sukzessiven Prozess hin.

\section{Literatur}

Albrecht, M. (1997): ARD-digital: Vernetzen statt Versparten. Das digitale Programmbouquet der ARD. In: Media Perspektiven 8/1997: 415-417.

Beisch, N. und B. Engel (2006): Wie viele Programme nutzen die Fernsehzuschauer? In: Media Perspektiven 7/2006: 374-379.

Blumler, J. G. (1979): The Role of Theory in Uses and Gratifications Studies. In: Communication Research 6(1): 9-36.

Brosius, F. (2004): SPSS 12. Bonn: mitp-Verlag.

Conway, J. und A. Rubin (1991): Psychological Predictors of Television Viewing Motivation. In: Communication Research 18(4): 443-463.

Eilders, C. (1999): Zum Konzept der Selektivität: Auswahlprozesse bei Medien und Publikum. In: W. Wirth und W. Schweiger (Hrsg.): Selektion im Internet. Empirische Analysen zu einem Schlüsselkonzept. Opladen: Westdeutscher Verlag: 13-42.

Engel, B. (2006): Digitales Fernsehen in Deutschland. Zwischen Fortschritt und Stagnation. In: W. Koschnik (Hrsg.): Focus-Jahrbuch 2006. Schwerpunkt Lifestyle Forschung. München: Focus Magazin Verlag: 449-463.

Ferguson, D. A. und E. M. Perse (1993): Media and Audience Influences on Channel Repertoire. In: Journal of Broadcasting \& Electronic Media 37(1): 31-47.

Hans-Bredow-Institut (Hrsg.) (2005): DocuWatch Digitales Fernsehen. Eine Sichtung ausgewählter Dokumente und wissenschaftlicher Studien. Themenheft: Electronic Programme Guides (EPGs). Heft 2/2005. Hamburg: Verlag Hans-Bredow-Institut. http://www.hans-bredowinstitut.de/publikationen/dw/2005/docuwatch_2-2005.pdf (3.5.2007). 
Hasebrink, U. (2001): Fernsehen in neuen Medienumgebungen. Befunde und Prognosen zur Zukunft der Fernsehnutzung. Berlin: Vistas Verlag.

Hasebrink, U. (2003): Nutzungsforschung. In: G. Bentele, H.-B. Brosius und O. Jarren (Hrsg.): Öffentliche Kommunikation. Wiesbaden: Westdeutscher Verlag: 101-127.

Heeter, C. (1985): Program Selection with Abundance of Choice: A Process Modell. In: Human Communication Research 12(1): 126-152.

Heeter, C. und B. Greenberg (1988): A Theoretical Overview of the Program Choice Process. In: C. Heeter und B. Greenberg (Hrsg.): Cableviewing. Norwood: Ablex Publishing Company: 33-50.

Kang, M.-H. (2002): Interactivity in Television. Use and Impact of an Interactive Program Guide. In: Journal of Broadcasting and Electronic Media 46(3): 330-345.

Leopoldt, S. (2002): Navigatoren. Zugangsregulierung bei elektronischen Programmführern im digitalen Fernsehen. Baden-Baden: Nomos Verlagsgesellschaft.

Levy, M. R. und S. Windahl (1984): Audience Activity and Gratifications. A Conceptual Clarification and Exploration. In: Communication Research 11(1): 51-78.

Levy, M. R. und S. Windahl (1985): The Concept of Audience Activity. In: K. E. Rosengren, L. A. Wenner und P. Palmgreen (Hrsg.): Media Gratifications Research. Beverly Hills, London, New Delhi: Sage Publications: 109-122.

Litz, H. P. (2000): Multivariate Statistische Methoden und ihre Anwendung in den Wirtschafts- und Sozialwissenschaften. München, Wien: Oldenbourg Wissenschaftsverlag.

Mansmann, T. (2005): Elektronische Guides ergänzen Gedrucktes. In: Horizont 44/2005 vom 3.11.2005: 64.

McQuail, D. (1997): Audience Analysis. Thousand Oaks, London, New Delhi: Sage Publications.

Meyen, M. (2004): Mediennutzung - Medienforschung, Medienfunktionen, Nutzungsmuster. Konstanz: UVK Verlagsgesellschaft.

Niemeyer, H.-G. und J. M. Czycholl (1994): Zapper, Sticker und andere Medientypen. Stuttgart: Schäffer-Poeschel Verlag.

Paus-Hasebrink, I. (2005): Zur Implementierung von DVB-T in Österreich. Eine qualitative Studie. Wien.http://www.rtr.at/web.nsf/deutsch/Foerderungen_Digitalisierungsfonds_Berichte/\$file/ Studie_Universitaet_Salzburg.pdf (3.5.2007).

Rubin, A. M. (1981): An Examination of Television Viewing Motivations. In: Communication Research 8(2): 141-165.

Rubin, A. M. (1983): Television Uses and Gratifications: The Interactions of Viewing Patterns and Motivations. In: Journal of Broadcasting 27(1): 37-51.

Rubin, A. M. (1984): Ritualized and Instrumental Television Viewing. In: Journal of Communication 34(3): 67-77.

Rubin, A. M. (2002): The Uses-and-Gratifications-Perspective of Media Effects. In: J. Byrant und D. Zillmann (Hrsg.): Media Effects. Advances in Theory and Research. Mahwah, London: Lawrence Erlbaum Associates: 525-548.

Ruggiero, T. E. (2000): Uses and Gratifications Theory in the 21st Century. In: Mass Communication \& Society 3(1): 3-37.

Schenk, M. (2002): Medienwirkungsforschung. Tübingen: Mohr Verlag.

Schenk, M., T. Döbler und B. Stark (2002): Marktchancen des digitalen Fernsehens. Wiesbaden: Westdeutscher Verlag.

Seemann, W. (2005): Konkurrent Computer. In: werben \& verkaufen Nr. 10 vom 9.3.2006: 64.

Stark, B. (2006): Fernsehen in digitalen Medienumgebungen. Eine empirische Analyse des Zuschauerverhaltens. München: Verlag Reinhard Fischer.

Stipp, H. (2003): Entwicklung digitaler Fernsehtechniken in den USA. In: Media Perspektiven 10/2003: 470-476.

Stipp, H. (2004): Die Fernsehentwicklung in den USA - 10 Jahre danach. In: Media Perspektiven 12/2004: 569-575.

Webster, J. G. und J. J. Wakshlag (1983): A Theory of Television Program Choice. In: Communication Research 10(4): 430-446. 
M\&K 55. Jahrgang 2/2007

Winterhoff-Spurk, P. (1999): Medienpsychologie. Eine Einführung. Stuttgart, Berlin, Köln: Kohlhammer.

Zubayr, C. und H. Gerhard (2007): Tendenzen im Zuschauerverhalten. In: Media Perspektiven 4/2007: 187-199. 